\title{
The functional Hfq-binding module of bacterial sRNAs consists of a double or single hairpin preceded by a U-rich sequence and followed by a $3^{\prime}$ poly $(U)$ tail
}

\author{
HIROKAZU ISHIKAWA, ${ }^{1}$ HIRONORI OTAKA, ${ }^{1}$ KIMIKA MAKI, ${ }^{1}$ TEPPEI MORITA, ${ }^{2}$ and HIROJI AIBA ${ }^{2,3}$ \\ ${ }^{1}$ Division of Biological Science, Graduate School of Science, Nagoya University, Chikusa, Nagoya 464-8602, Japan \\ ${ }^{2}$ Faculty of Pharmaceutical Sciences, Suzuka University of Medical Sciences, Suzuka, Mie 513-0816, Japan
}

\begin{abstract}
Hfq-dependent sRNAs contain, at least, an mRNA base-pairing region, an Hfq-binding site, and a Rho-independent terminator. Recently, we found that the terminator poly $(U)$ of Escherichia coli sRNAs is essential for Hfq binding and therefore for riboregulation. In this study, we tried to identify additional components within Hfq-binding sRNAs required for efficient Hfq binding by using SgrS as a model. We demonstrate by mutational and biochemical studies that an internal hairpin and an immediately upstream $\mathrm{U}$-rich sequence also are required for efficient $\mathrm{Hfq}$ binding. We propose that the functional $\mathrm{Hfq}$-binding module of SgrS consists of an internal hairpin preceded by a U-rich sequence and a Rho-independent terminator with a long $\operatorname{poly}(U)$ tail. We also show that the Rho-independent terminator alone can act as a functional Hfq-binding module when it is preceded by an internal U-rich sequence. The 3' region of most known sRNAs share the features corresponding to either a doubleor single-hairpin-type $\mathrm{Hfq}$-binding module. We also demonstrate that increasing the spacing between the base-pairing region and the Hfq-binding module reduces or impairs the silencing ability. These findings allowed us to design synthetic Hfq-binding sRNAs to target desired mRNAs.
\end{abstract}

Keywords: Hfq; bacterial sRNA; Rho-independent terminator; internal hairpin; U-rich sequence

\section{INTRODUCTION}

The RNA chaperone Hfq is essential for the regulatory function of trans-acting Hfq-binding small RNAs (sRNAs) at a post-transcriptional level in enteric bacteria. The functional roles of $\mathrm{Hfq}$ in sRNA action are fairly well understood. The primary role of $\mathrm{Hfq}$ is to facilitate basepairing between sRNAs and target mRNAs to regulate their translation, mostly negatively and also positively in some cases (Gottesman and Storz 2010; Vogel and Luisi 2011). Another role of Hfq is to recruit RNase E near target mRNAs to destabilize sRNA-mRNA hybrids (Morita et al. 2005). In addition, $\mathrm{Hfq}$ is known to stabilize sRNAs by protecting them from the attack of ribonucleases (Gottesman 2004; Storz and Gottesman 2006). The identification of Hfq-binding sites on sRNAs and mRNAs is crucial to

\footnotetext{
${ }^{3}$ Corresponding author.

E-mail aiba@suzuka-u.ac.jp.

Article published online ahead of print. Article and publication date are at http://www.rnajournal.org/cgi/doi/10.1261/rna.031575.111.
}

understand the mechanism by which Hfq facilitates the base-pairing.

Hfq-dependent sRNAs contain at least three functional elements, an mRNA base-pairing region, an Hfq-binding site, and a Rho-independent transcription terminator. The three elements of sRNAs can be located in different regions of sRNAs, or they can structurally overlap. The base-pairing region that is partially complementary to the target mRNA is well defined in many sRNAs. The role of the base-pairing region is to form an RNA-RNA hybrid with the target mRNA to regulate its translation and stability. The Rhoindependent terminator, characterized as a GC-rich palindrome sequence followed by a run of $U$ residues, is also clearly defined. The primary role of the Rho-independent terminator of sRNAs is to terminate transcription, resulting in distinct sRNA molecules. Another important role of the terminator hairpin is to stabilize the transcribed RNAs (Aiba et al. 1991). The Hfq-binding site is essential for Hfq to facilitate the pairing between a given sRNA and the target mRNA. While the functional role of the Hfq-binding site is obvious, the exact entity and/or structural composition of functional Hfq site are only partially understood. 
Hfq forms a donut-shaped homohexameric ring structure through the N-terminal Sm domain (Moller et al. 2002; Schumacher et al. 2002; Zhang et al. 2002). Structural studies on Hfq-RNA oligonucleotide complexes revealed that one side of the Hfq hexamer, called the proximal side, binds U-rich sequences, while the other side, called the distal side, binds A-rich sequences or A-R-N repeats (Schumacher et al. 2002; Link et al. 2009; Sauer and Weichenrieder 2011). In accordance with these structural studies, biochemical studies demonstrated that $\mathrm{Hfq}$ binds preferentially AU-rich sequences in several sRNAs such as OxyS (Zhang et al. 2002), Spot42 (Moller et al. 2002), DsrA (Brescia et al. 2003), RyhB (Geissmann and Touati 2004), MicA (Rasmussen et al. 2005), and RybB (Balbontin et al. 2010). Most of these AU-rich sequences are located internally in sRNA molecules near stem-loop structures. However, little mutational analysis of the Hfq-binding sites has been performed in these cases. Thus, it remains unclear whether or not the physically identified Hfq-binding sites are really needed for Hfq action. Recently, we demonstrated that the Rhoindependent terminators of Hfq-binding sRNAs posses more than seven consecutive $U$ residues in general, and this long terminator poly $(\mathrm{U})$ tail is essential for Hfq binding and therefore for riboregulation (Otaka et al. 2011). Structural and biochemical studies by another group have shown that Hfq preferentially binds U-rich sequences of the $3^{\prime}$ end of RNA molecules in vitro (Sauer and Weichenrieder 2011). An important question is whether the terminator poly(U) tail alone is sufficient for efficient Hfq-sRNA interaction. The aim of this study is to answer this question and to define the structural composition required for the functional $\mathrm{Hfq}$ binding within sRNAs by using SgrS as a model.

SgrS is one of the well-characterized Hfq-binding sRNAs that is induced under glucose-phosphate stress in Escherichia coli (Vanderpool and Gottesman 2004). It binds to the translational initiation region of target $p t s G$ mRNA, encoding the membrane component of the major glucose transporter, resulting in translational repression and RNase E-dependent rapid degradation of $p t s G$ mRNA (Aiba 2007). Here, we show that the terminator poly(U) tail of SgrS alone is not sufficient for efficient interaction with Hfq. Fine mutational and biochemical analyses revealed that the 3 ' region of SgrS consisting of an internal hairpin structure preceded by a U-rich sequence and the terminator hairpin followed by a long poly(U) tail are the functional Hfabinding module of SgrS. The terminator hairpin preceded by a U-rich sequence and followed by a long poly(U) tail can act as the simplest form of a functional Hfq-binding module. In addition, we showed that increasing spacing between the Hfq-binding module and the base-pairing region reduces or impairs the silencing ability. Finally, we were able to design synthetic Hfq-binding sRNAs successfully to target desired mRNAs based on the present findings.

\section{RESULTS}

\section{An internal hairpin is important for SgrS function}

We showed previously that SgrS14 carrying mutations at nucleotides 183-187 loses the ability to silence the target pts $G$ mRNA (Maki et al. 2010). The nucleotides 183-187 of SgrS were expected to be involved in base-pairing with $p t s G$ mRNA because they are complementary to nucleotides 80-76 of $p t s G$ mRNA (Fig. 1A). However, further mutational study established that the pairing between these regions is dispensable for SgrS function, suggesting that the 183-187 region contributes to the SgrS function by other means (Maki et al. 2010). We noticed that nucleotides 183-186 of SgrS potentially base-pair with nucleotides 193-196 of the same SgrS molecule, forming a stem-loop structure (Fig. $1 \mathrm{~A}, \mathrm{~B})$. In addition, a U-rich sequence, UAUU, exists immediately upstream of this potential hairpin structure. Because Hfq is known to bind preferentially AU-rich sequences near hairpin structures in several sRNAs (Moller et al. 2002; Zhang et al. 2002; Brescia et al. 2003; Geissmann and Touati 2004; Rasmussen et al. 2005; Balbontin et al. 2010), this hairpin structure along with the preceding U-rich sequence could be involved in SgrS function by acting as an additional Hfq-binding site. To test this possibility, compensatory mutations to retain the intramolecular pairing were introduced in nucleotides 192-196 of SgrS14. Then, we examined the silencing ability of the resulting SgrS14R (Fig. 1C) compared with SgrS and SgrS14. First, each mutated sgrS gene was placed under the control of an IPTG-inducible promoter on plasmid pQE80L. Each plasmid was introduced into $h f q^{+}$and $\Delta h f q$ cells. The expression of RNAs and $\mathrm{IICB}^{\mathrm{Glc}}$, the protein product of $p t s G$ mRNA, was analyzed by Northern and Western blotting, respectively (Fig. 2A). We confirmed the previous finding (Maki et al. 2010) that SgrS14 fails to silence $p t s G$ mRNA, while the wild-type SgrS strongly down-regulates the expression of $p t s G$ mRNA in an $h f q^{+}$background (Fig. 2A, lanes 2,3). Interestingly, SgrS14R restores the ability to silence $p t s G \mathrm{mRNA}$ (Fig. 2A, lane 4), indicating that the internal hairpin is important for the silencing function of SgrS. The expression level of hairpindisrupting mutant SgrS14 is moderately lower than that of wild-type SgrS in an $h \mathrm{fq}^{+}$background (Fig. 2A, lanes 2,3), suggesting that the internal hairpin structure is somehow involved in Hfq binding. In addition, the levels of $p t s G$ mRNA and IICB $^{\text {Glc }}$ consistently increase in a $\Delta h f q$ background, suggesting that $\mathrm{Hfq}$ moderately affects transcription and/or stability of the ptsG mRNA in an SgrS-independent manner.

SgrS14 carries base substitutions not only at nucleotides 183-187 but also at several positions upstream of the minimal base-pairing region (Fig. 1C). To test further the role of the internal stem-loop in SgrS function, we constructed two additional hairpin-disrupting mutants, SgrS14a and SgrS14b, in which only the region corresponding to one 
A

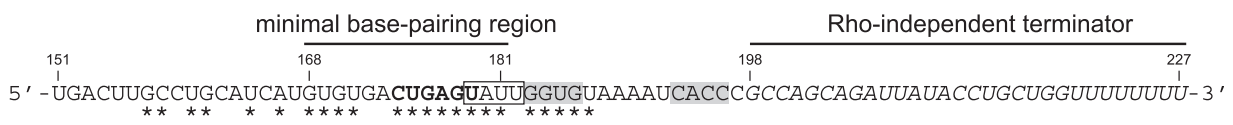

B

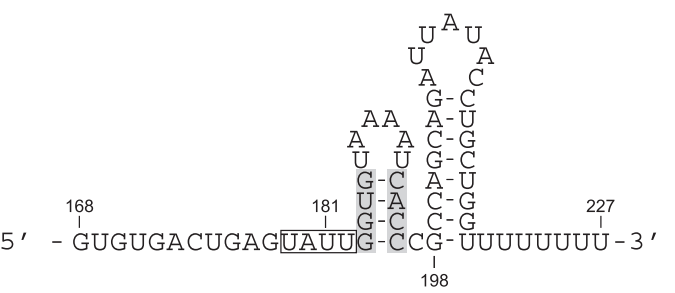

C

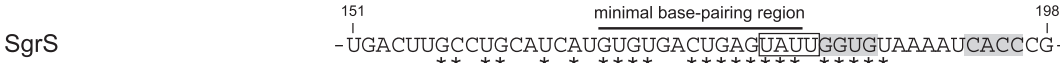

$\begin{array}{cccc}\nabla \nabla & \nabla & \nabla & \nabla \\ \text { SgrS14 UGACUUUGCAUCAACUUGUGUGACUGAGUAUUCCACGAAAAUCACCCG- } & \nabla \nabla \nabla \nabla\end{array}$

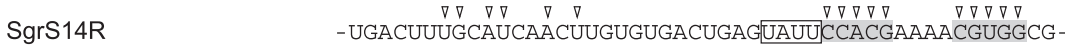

$\begin{array}{ll}\text { SgrS14a } & \text {-UGACUUGCCUGCAUCAUGUGUGACUGAGUAUUCCACGAAAAUCACCCG } \\ & \nabla \nabla \nabla \nabla\end{array}$

SgrS14aR -UGACUUGCCUGCAUCAUGUGUGACUGAGUAUUCCACGAAAACGUGGCG-

SgrS14b -UGACUUGCCUGCAUCAUGUGUGACUGAGUAUUUAGCAAAAAUCACCCG-

SgrS14bR -UGACUUGCCUGCAUCAUGUGUGACUGAGUAUUUAGCAAAAAUGCUACG-

SgrS-LGC -UGACUUGCCUGCAUCAUGUGUGACUGAGUAUUGGUGGAGCACCACCCG -

SgrS-182C -UGACUUGCCUGCAUCAUGUGUGACUGAGUAUCGGUGUAAAAUCACCCG-

SgrS-CCGC -UGACUUGCCUGCAUCAUGUGUGACUGAGCCGCGGUGUAAAAUCACCCG-

SgrSi

SgrSiCCGC

SgrSiUUUU

SgrSiAAAA

SgrS14aUUUU

SgrS14aAAAA

SgrS14aCcCC

$\nabla \nabla \nabla$
-UGACUUGCCUGCAUCAUGUGUGACUGAGUAUUAUUGGUGUAAAAUCACCCG-

$\nabla \nabla \nabla \nabla \nabla \nabla \nabla$

- UGACUUGCCUGCAUCAUGUGUGACUGAGUAUCCGCGGUGUAAAAUCACCCG-

$\nabla \nabla \nabla \nabla \nabla \nabla \nabla$

- UGACUUGCCUGCAUCAUGUGUGACUGAGUAUUUUUUGGUGUAAAAUCACCCG-

$\nabla \nabla \nabla \nabla \nabla \nabla \nabla$

- UGACUUGCCUGCAUCAUGUGUGACUGAGUAUAAAAGGUGUAAAAUCACCCG-

$\nabla \nabla \nabla \nabla \nabla$
- UGACUUGCCUGCAUCAUGUGUGACUGAGUAUUCCACGAAAAUUUUUCG -

- UGACUUGCCUGCAUCAUGUGUGACUGAGUAUUCCACGAAAAUAAAACG -

$\nabla \nabla \nabla \nabla \nabla \quad \nabla \nabla \nabla \nabla$

SgrS $\triangle 5$ - UGACUUGCCUGCAUCAUGUGUGACUGAGUAUU- - - - - AAAAUCACCCG-

SgrS $\triangle 10 \quad$ - UGACUUGCCUGCAUCAUGUGUGACUGAGUAUU - - - - - - - CACCCG-

SgrS $\triangle 14 \quad$ - UGACUUGCCUGCAUCAUGUGUGACUGAGUAUU- - . - . - - - - - CG-

FIGURE 1. (A) The nucleotide sequence of the $3^{\prime}$ region of SgrS. The $3^{\prime}$ portion of SgrS consists of the minimal base-pairing region (168-181) (Maki et al. 2010), a terminator hairpin including a poly(U) stretch (198-227), and a spacer (182-197). $\left(^{*}\right.$ ) Nucleotides complementary to the translation initiation region of ptsG mRNA (Vanderpool and Gottesman 2004). The bold letters represent the crucial nucleotides for SgrS action (Kawamoto et al. 2006). The inverted repeat sequences in the spacer region are shadowed. The UAUU sequence before the potential hairpin structure is boxed. The Rho-independent terminators are italicized. (B) The predicted secondary structure of the 3' portion of SgrS. (C) The nucleotide sequences of the $3^{\prime}$ portion of SgrS variants. (Arrowheads) Nucleotides changed from the wild-type SgrS sequence. The inverted repeat sequences in the spacer region are shadowed. The U-rich sequences before internal or terminator hairpins are boxed.

strand of the internal hairpin stem is mutated (Fig. 1C). We also constructed compensatory mutants, SgrS14aR and SgrS14bR, that restore the intramolecular pairing. Then, we examined the silencing ability of each SgrS variant exressed from the corresponding gene under an arabinose-inducible promoter on the low-copy-number plasmid in $h f q^{+}$cells.
SgrS14a and SgrS14b failed to silence ptsG mRNA, while SgrS14aR and SgrS14bR regained the silencing ability (Fig. 2B, lanes 3-6). The hairpin-disrupting SgrS variants were also expressed from the corresponding genes under an arabinose-inducible promoter on the multicopy plasmid in $h \mathrm{fq}^{+}$cells. Interestingly, SgrS14a and SgrS14b were able to 
A

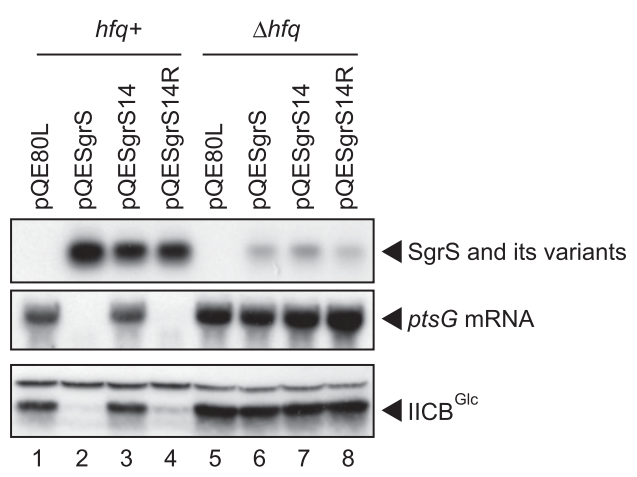

B

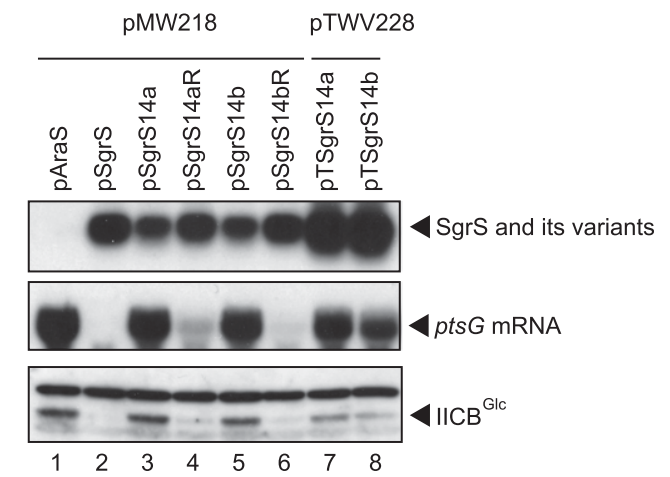

FIGURE 2. Effects of mutations in internal stem-loop on expression and silencing ability of SgrS. (A) Properties of SgrS variants expressed from pQE80L-series plasmids. IT1568 $\left(h f q^{+}\right)$and TM589 $(\Delta h f q)$ cells harboring indicated plasmids were grown in $\mathrm{LB}$ medium in the presence of $0.1 \mathrm{mM}$ IPTG. Total RNAs and proteins were prepared. The RNA samples were subjected to Northern blot analysis using SgrS probe 1 and a pts $G$ probe. The following amounts of RNAs were loaded: SgrS variants, $0.1 \mu \mathrm{g} ; \mathrm{pts} G \mathrm{mRNA}, 10 \mu \mathrm{g}$. Protein samples equivalent to $0.04 \mathrm{~A}_{600}$ units were subjected to Western blot analysis. $(B)$ Properties of SgrS variants expressed from pMW218- and pTWV228-series plasmids. pMW218 is a low-copy, and pTWV228 is a high-copy plasmid. IT1568 cells harboring indicated plasmids were grown in the presence of $0.2 \%$ (for preparation of total RNAs) or $1 \%$ (for preparation of total proteins) arabinose. Total RNAs and proteins were prepared. RNA samples were subjected to Northern blot analysis using SgrS probe 1 and a pts $G$ probe. The following amounts of RNAs were loaded: SgrS variants, $0.25 \mu \mathrm{g}$; pts $G$ mRNA, $10 \mu \mathrm{g}$. Protein samples equivalent to $0.04 \mathrm{~A}_{600}$ units were subjected to Western blot analysis.

repress significantly the expression of $p t s G$ mRNA when their abundance was elevated (Fig. 2B, lanes 7,8). We also confirmed again the previous finding (Otaka et al. 2011) that SgrSLS4U possessing a shortened terminator poly $(\mathrm{U})$ tail along with an extended terminator stem completely fails to silence $p t s G$ mRNA despite its higher abundance (data not shown). Taken together, we conclude that the stem-loop in the 183-196 region surely contributes to the efficient action of SgrS, but its requirement is not as strict as that of the terminator $\operatorname{poly}(\mathrm{U})$ tail.

\section{U-rich sequence near the stem-loop is also involved in SgrS function}

Two AU-rich sequences exist near the internal stem-loop, UAUU from nucleotides 179-182 and UAAAAU from nucleotides 187-192 (Fig. 1A). To examine whether these AU-rich sequences are involved in Hfq-dependent SgrS function, we have introduced mutations in these sites and tested their effects on SgrS function. First, the UAAAAU sequence in the predicted hairpin loop was converted to GAGCAC. The resulting SgrSLGC (Fig. 1C) retains the full Hfqdependent silencing ability (Fig. 3A, lane 3), indicating that the UAAAAU sequence in the loop is dispensable for SgrS function. Then, we examined the effect of mutations in the UAUU sequence from nucleotides 179-182. Because this sequence except U182 overlaps with the minimal basepairing region, we first constructed SgrS182C carrying a U-to-C substitution at nucleotide 182 (Fig. 1C) and examined its behavior. SgrS182C exhibited a reduced ability to silence
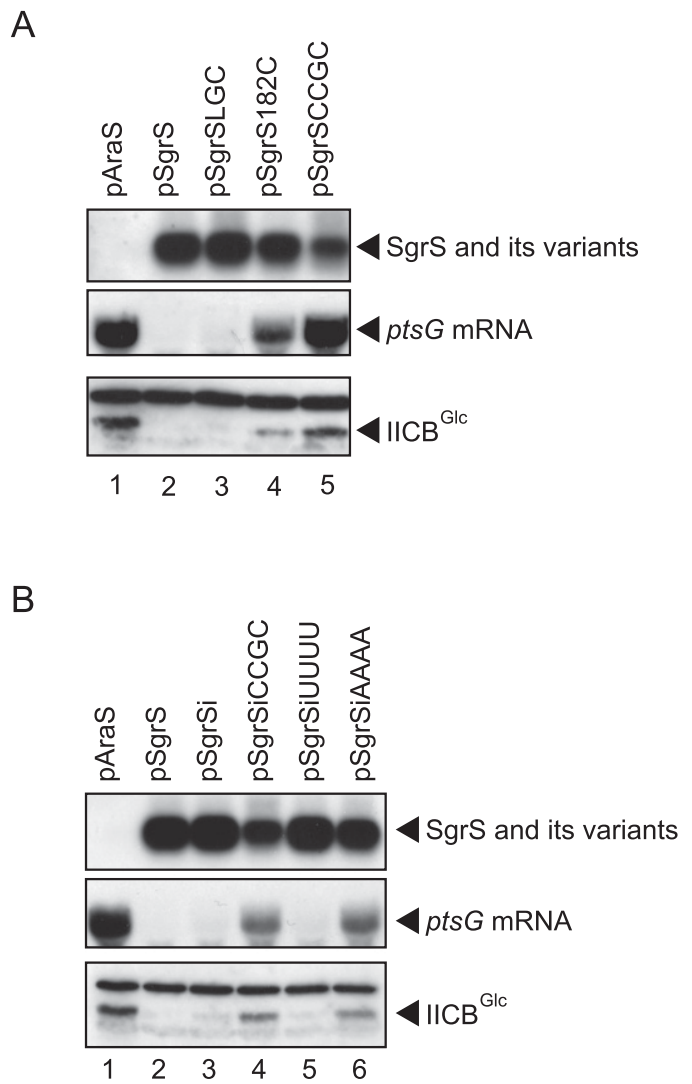

FIGURE 3. Effects of mutations in AU-rich sequences on expression and silencing ability of SgrS. Properties of SgrS-series $(A)$ and SgrSiseries $(B)$ variants were examined. IT1568 cells harboring indicated plasmids were grown in the presence of $0.2 \%$ (for preparation of total RNAs) or $1 \%$ (for preparation of total proteins) arabinose. Total RNAs and proteins were prepared. RNA samples were subjected to Northern blot analysis using SgrS probe 1 and a $p t s G$ probe. The following amounts of RNAs were loaded: SgrS variants, $0.25 \mu \mathrm{g}$; $p t s G$ mRNA, $10 \mu \mathrm{g}$. Protein samples equivalent to $0.04 \mathrm{~A}_{600}$ units were subjected to Western blot analysis. 
the ptsG mRNA, suggesting that the UAUU sequence is somehow involved in SgrS function (Fig. 3A, lane 4). Then, we converted the UAUU sequence to CCGC. The resulting SgrSCCGC (Fig. 1C) is inactive regarding the silencing ability (Fig. 3A, lane 5). This is as expected because the first $3 \mathrm{nt}$ of the UAUU sequence are also components of the minimal base-pairing region (Fig. 1A). To examine the effect of mutations in the UAUU sequence on SgrS function without losing the base-pairing ability, we constructed another SgrS variant, SgrSi, in which UAU is inserted before the UAUU sequence (Fig. 1C). The insertion of the UAU did not affect the expression and the silencing ability of SgrS (Fig. 3B, lane 3). Then, we converted the UAUU sequence to CCGC, UUUU, or AAAA (Fig. 1C), and examined the activity of the resulting SgrS variants. Interestingly, SgrSiCCGC and SgrSiAAAA exhibited a reduced silencing ability, while SgrSiUUUU retains the full silencing ability (Fig. 3B, lanes 4-6). These results suggest that the UAUU sequence is required, although not absolutely, for SgrS function. It is also clear that the U-rich but not A-rich property near the stem-loop is important for SgrS function.

\section{The internal hairpin and the immediately upstream $\mathrm{U}$-rich sequence are involved in $\mathrm{Hfq}$ binding}

The results mentioned above suggest that the internal hairpin and the preceding U-rich sequence within SgrS are involved in Hfq binding. To examine the Hfq-binding ability of SgrS variants in vivo, we performed a pull-down assay using cells in which Hfq-Flag and each SgrS variant were expressed from the chromosomal gene and the corresponding gene on the low-copy-plasmid, respectively. Cell extracts were incubated with anti-Flag M2-agarose beads. Proteins bound to the agarose beads were analyzed by Western blotting using anti-Flag antibodies. The affinity-purified Hfq-Flag was treated with phenol and subjected to Northern blotting. As reported previously (Otaka et al. 2011), SgrSLS4U, a mutant with an extended terminator stem and a shortened terminator $U$ stretch, fails to coimmunoprecipitate with Hfq-Flag, while wild-type SgrS efficiently binds Hfq (Fig. $4 \mathrm{~A}$, lanes 6,10). Then, we examined the effect of hairpindisrupting mutations on Hfq binding, focusing on SgrS14b and SgrS14bR. SgrS14b failed to coimmunoprecipitate with Hfq-Flag (Fig. 4A, lane 7), suggesting that disruption of the internal hairpin impairs Hfq binding. One could argue that the failure of coimmunoprecipitation is due to the reduced abundance of SgrS14b in crude extract compared with SgrS (Fig. 4A, lane 2). Importantly, SgrS14bR, the suppressor mutant of SgrS14b that restores intramolecular pairing, also restored the ability to bind $\mathrm{Hfq}$ (Fig. 4A, lane 8), although it is similar in abundance to SgrS14b. Thus, the data clearly indicate that the internal stem-loop is required for efficient $\mathrm{Hfq}$ binding of SgrS. We also examined the effect of mutation in the U-rich sequence on Hfq binding using SgrSCCGC. The Hfq-binding ability of SgrSCCGC is
A

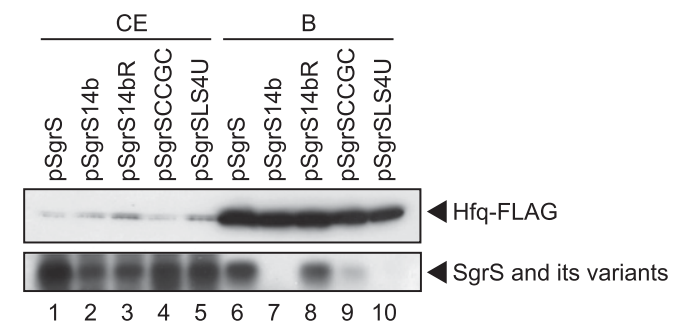

B

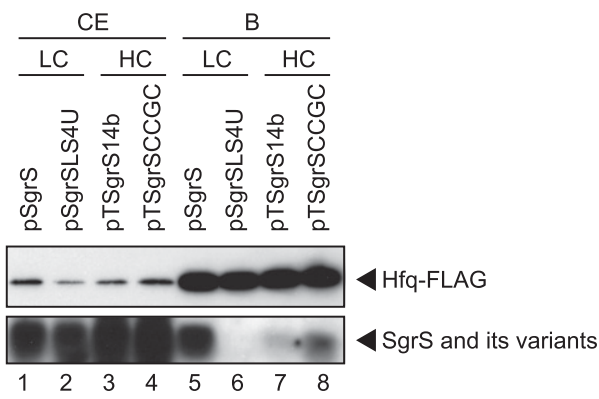

FIGURE 4. Effects of mutations on Hfq binding in vivo. (A) Hfq binding of SgrS variants expressed from the low-copy plasmid. (B) Hfq binding of SgrS variants expressed from the high-copy plasmid. Crude extracts were prepared from TM771 ( $\Delta$ sgrS $h f q-F L A G$-cat $)$ cells harboring indicated plasmids and subjected to the pull-down assay using anti-Flag agarose as described in Materials and Methods. Crude extracts $(\mathrm{CE} ; 5 \mu \mathrm{L})$ and bound fractions $(\mathrm{B} ; 5 \mu \mathrm{L})$ were analyzed by Western blotting using anti-Flag antibodies. For analysis of RNAs, crude extracts $(3 \mu \mathrm{L})$ and bound fractions $(3 \mu \mathrm{L})$ were treated with phenol and subjected to Northern blotting using SgrS probe 1.

markedly reduced (Fig. 4A, lane 9), suggesting that the $\mathrm{U}$-rich sequence is also required for efficient Hfq binding of SgrS. Taken together, we conclude that, in addition to the terminator $\operatorname{poly}(\mathrm{U})$ tail, the internal stem-loop and the immediately upstream U-rich sequence are needed for efficient Hfq binding. We propose that the whole region downstream from nucleotide 179 corresponds to the functional Hfq-binding module of SgrS. This region consists of an internal U-rich sequence followed by a stem-loop and a Rho-independent terminator with a long poly(U) tail.

It should be noted that a small amount of SgrSCCGC coimmunoprecipitated with Hfq-Flag even when expressed using the low-copy plasmid (Fig. 4A, lane 9), suggesting that the Hfq-binding ability of SgrSCCGC is reduced but not completely eliminated. To examine further the Hfqbinding ability of SgrSCCGC and SgrS14b, these SgrS variants were expressed using the high-copy plasmid, and the pull-down assay was performed. A significant amount of SgrSCCGC coimmunoprecipitated with Hfq-Flag (Fig. $4 \mathrm{~B}$, lane 8), indicating that SgrSCCGC retains the Hfqbinding ability to some extent. SgrS14b also coimmunoprecipitated, though weakly, with Hfq-Flag when expressed using the high-copy plasmid (Fig. 4B, lane 7), suggesting that SgrS14b also retains a weak Hfq-binding ability. 


\section{Hfq binding of SgrS variants in vitro}

The mutational and biochemical analyses indicate that poly $(\mathrm{U})$ shortening eliminates the Hfq binding of SgrS, while disruption of either the internal stem-loop or U-rich sequence significantly reduces Hfq-binding in vivo. To test directly Hfq binding of SgrS variants in vitro, we investigated the interaction between SgrS variants prepared by in vitro transcription and purified $\mathrm{Hfq}-\mathrm{His}_{6}$ by gel mobility shift assay. First, a fixed amount of ${ }^{32} \mathrm{P}$-labeled wild-type SgrS RNA was incubated with increasing concentrations of $\mathrm{Hfq}_{-} \mathrm{His}_{6}$. The complex formation was analyzed by gel electrophoresis on a native polyacrylamide gel. When the ratio of $\mathrm{Hfq}$ hexamer to SgrS was $<1$, a single retarded complex I along with free ${ }^{32} \mathrm{P}$-labeled SgrS was observed (Fig. 5A, lane 2). When equal amounts of Hfq hexamer and ${ }^{32} \mathrm{P}$-labeled SgrS were incubated together, essentially all of the ${ }^{32} \mathrm{P}$-labeled SgrS formed complex I (Fig. 5A, lane 3). When the amount of $\mathrm{Hfq}^{-\mathrm{His}_{6}}$ was increased further, complex I was still observed as a major band, although additional retarded complexes of higher Hfq-to-SgrS ratios were also formed (Fig. 5A, lanes 4-7). These results suggest that Hfq hexamer and SgrS form predominantly the stable 1:1 complex I under our experimental conditions, although we do not exclude the possibility that Hfq binds SgrS in other stoichiometries.

Then, we examined the effect of excess amounts of unlabeled SgrS variants on the formation of ${ }^{32} \mathrm{P}$-labeled SgrS RNA/Hfq complex I. When excess amounts of unlabeled wild-type SgrS were mixed together, the formation of complex I markedly decreased as expected (Fig. 5B, lanes 3,4). The same amount of SgrS4U, a mutant with a shortened terminator U stretch (Otaka et al. 2011), did not affect the complex formation between ${ }^{32} \mathrm{P}$-labeled wild-type SgrS RNA and $\mathrm{Hfq}$ at all (Fig. 5B, lanes 5,6), indicating that the Hfq-binding ability of SgrS4U is heavily impaired. This result is perfectly consistent with our previous finding that SgrS4U and SgrSLS4U lose the ability to bind Hfq in vivo (Otaka et al. 2011). The same competition experiments were performed by using excess amounts of other variants. SgrS14b and SgrSCCGC inhibited the SgrS-Hfq interaction to some extent, although their effects are clearly weaker than that of wild-type SgrS (Fig. 5B, lanes 7,8,11,12). Thus, these two variants still partially retain Hfq-binding ability in vitro. The results also show that the Hfq-binding ability of SgrSCCGC is apparently higher than that of SgrS14b. As expected, SgrS14bR inhibits the SgrS-Hfq interaction efficiently (Fig. 5B, lanes 9,10), indicating that the Hfqbinding ability is restored to the wild-type level. The data indicate that the relative Hfq-binding ability of SgrS variants decreases in the following order: SgrS $=$ SgrS14bR $>$ SgrSCCGC $>$ SgrS14b $>$ SgrS4U. These in vitro binding data are consistent with the results of the in vivo binding study shown in Figure 4. Thus, it is apparent that the silencing ability of SgrS variants correlates with Hfq-binding ability. We conclude that the internal hairpin along with the U-rich
A

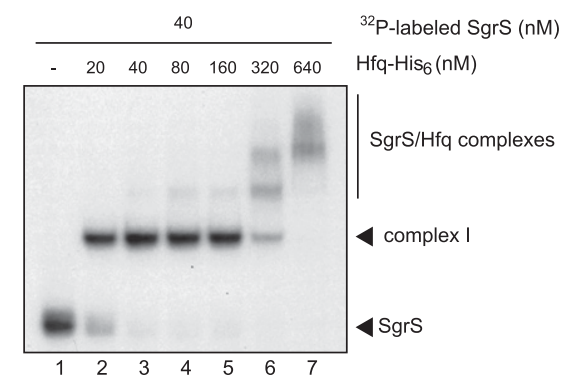

B

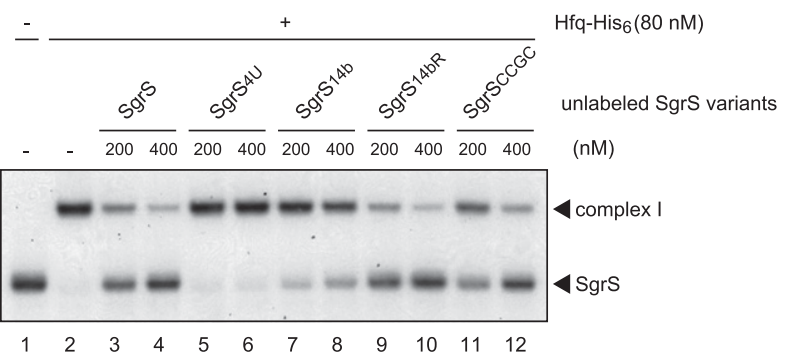

FIGURE 5. Hfq binding of SgrS variants in vitro. (A) Analysis of interaction between SgrS RNA and Hfq. The ${ }^{32} \mathrm{P}$-labeled SgrS RNA $(0.2 \mathrm{pmol})$ was incubated with increasing amounts of purified Hfq$\mathrm{His}_{6}$ in $5 \mu \mathrm{L}$ of binding buffer for $15 \mathrm{~min}$ at $37^{\circ} \mathrm{C}$. Samples were analyzed by a gel mobility shift assay after the addition of $5 \mu \mathrm{L}$ of RNA loading buffer. (B) Effects of SgrS variants on SgrS-Hfq interaction. The ${ }^{32} \mathrm{P}$-labeled SgrS RNA $(0.2 \mathrm{pmol})$ along with indicated amounts of each of unlabeled SgrS variants were incubated with $0.4 \mathrm{pmol}$ of purified $\mathrm{Hfq}-\mathrm{His}_{6}$ in $5 \mu \mathrm{L}$ of binding buffer for $15 \mathrm{~min}$ at $37^{\circ} \mathrm{C}$. Samples were analyzed by a gel mobility shift assay after the addition of $5 \mu \mathrm{L}$ of RNA loading buffer.

sequence is required for efficient Hfq binding that is a prerequisite for the silencing function of SgrS.

\section{SgrS variants in which a U-rich sequence is placed adjacent to the terminator hairpin are functional}

The results mentioned above raised an intriguing question whether the Rho-independent terminator alone, without the internal hairpin, could act as an Hfq-binding site if a U-rich sequence is placed just before the terminator hairpin. To address this question, nucleotides 193-196 of SgrS14a were changed to UUUU to construct SgrS14aUUUU (Fig. 1C) and tested its nature. Interestingly, SgrS14aUUUU became active for the pts $G$ silencing, and its expression level increased in $h f q^{+}$but not $\Delta h f q$ backgrounds (Fig. 6A, lanes 3,6). When nucleotides 193-196 of SgrS14a were changed to AAAA or CCCC, the resulting SgrS14aAAAA and SgrS14aCCCC remain inactive (Fig. 6A, lanes 4,5). Thus, the Rho-independent terminator alone could act as an Hfqbinding site without the internal hairpin when a U-rich sequence was placed just upstream of the terminator hairpin. If so, SgrS $\Delta 14$, in which nucleotides $183-196$ of SgrS corresponding to the internal stem-loop structure were deleted (Fig. 1C), would be expected to be active regarding 
A

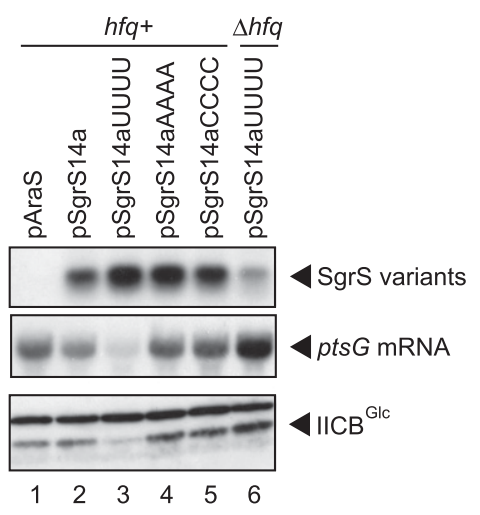

B

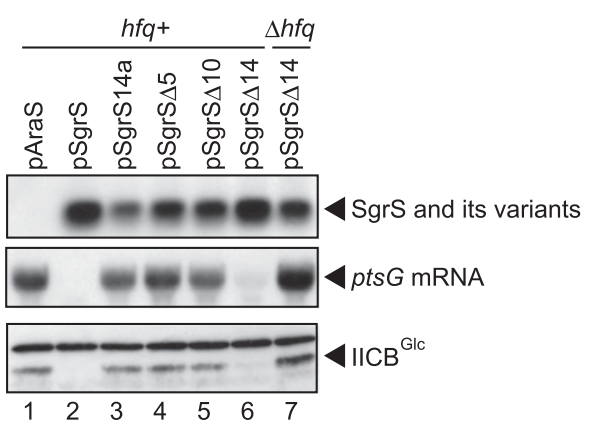

FIGURE 6. (A) Effects of nucleotide substitutions at positions before the terminator hairpin on properties of SgrS. (B) Effects of deletions in the spacer region on properties of SgrS. IT1568 and TM589 cells harboring indicated plasmids were grown in the presence of $0.2 \%$ (for preparation of total RNAs) or 1\% (for preparation of total proteins) arabinose. Total RNAs and proteins were prepared. RNA samples were subjected to Northern blot analysis using SgrS probe 1 and a $p t s G$ probe. The following amounts of RNAs were loaded: SgrS variants, $0.25 \mu \mathrm{g}$; pts $G \mathrm{mRNA}, 10 \mu \mathrm{g}$. Protein samples equivalent to $0.04 \mathrm{~A}_{600}$ units were subjected to Western blot analysis.

Hfq binding and the pts $G$ silencing because the UAUU sequence would then be just before the terminator hairpin in SgrS $\Delta 14$. In fact, SgrS $\Delta 14$ was able to efficiently silence the pts $G$ mRNA in $h f q^{+}$but not $\Delta h f q$ backgrounds (Fig. 6B, lanes $6,7)$. We conclude that the terminator hairpin followed by a long poly $(\mathrm{U})$ tail is sufficient for an Hfq-binding module when preceded by a U-rich sequence. We also constructed SgrS $\Delta 10$ and SgrS $\Delta 5$ in which nucleotides 183-192 and 183187 of SgrS14 are deleted, respectively (Fig. 1C). In these constructs, the UAUU sequence is separated by $5 \mathrm{nt}$ and $10 \mathrm{nt}$, respectively, from the terminator stem. Neither SgrS $\Delta 10$ nor $\operatorname{SgrS} \Delta 5$ was active for the $p t s G$ silencing (Fig. $6 \mathrm{~B}$, lanes $4,5)$. Thus, the U-rich sequence must be very close to the hairpin structure to act as a signal for Hfq binding.

\section{Effect of spacing between the Hfq-binding module and base-pairing region on SgrS function}

The finding that SgrS14aUUUU is active implies that the base-pairing region can be separated by at least 10 bases from the Hfq-binding module with keeping a significant silencing ability. Then, we addressed a question of how increasing the spacing between the base-pairing region and the Hfq-binding module affects the silencing ability of SgrS. For this, we constructed a series of spacing mutants by inserting different lengths of oligonucleotides up to $40 \mathrm{nt}$ between the base-pairing region and Hfq-binding module of SgrS-S (Fig. 7A). SgrS-S, a 5'-truncated SgrS variant, retains the full activity to down-regulate $p t s G$ mRNA, although it contains only nucleotides 168-227 of SgrS (Otaka et al. 2011). The inserted sequences are taken from the region of $g f p$ mRNA that is predicted to be unstructured. All of the inserted oligonucleotides possess a UAU sequence at their $5^{\prime}$ end. Therefore, these manipulations allowed us to separate physically the base-pairing region and the Hfq-binding module. The expression of SgrS-S variants and their silencing ability were tested by Northern and Western blotting. The data show that the insertion of up to $10 \mathrm{nt}$ does not affect the silencing ability (Fig. 7B, lanes 2-4), indicating that the Hfq-binding module can act efficiently when it is located within $10 \mathrm{nt}$ from the base-pairing region. Further increase of the spacing between two modules significantly decreases the silencing ability (Fig. 7B, lanes 5-7). When the Hfqbinding module was separated as much as $40 \mathrm{nt}$ away from the base-pairing region, the silencing ability was markedly reduced (Fig. 7B, lane 7). It is possible that the longer spacing mutants such as SgrS-S+40 are less efficient at riboregulation because the Hfq-binding module and/or the base-pairing region are sequestered in a secondary structure. The secondary structure prediction by the Mfold program (Zuker 2003) suggested that none of the inserted sequences does perturb the Hfq-binding module. In fact, we found that SgrS-S+40 and SgrS-S+20 coimmunoprecipitated as efficiently as SgrS-S (Fig. 7C). On the other hand, the inserted sequences in SgrS-S+10, SgrS-S+15, SgrS-S+20, and SgrS$S+40$ are predicted to base-pair with the base-pairing region in a similar manner. However, it is less likely that these potential pairings perturb the base-pairing region because SgrS-S+10 and SgrS-S+15 retain an efficient silencing ability. Thus, the simplest interpretation of the results of Figure 7B is that increased spacing impairs the Hfq-dependent basepairing ability of SgrS with the target mRNA without affecting the Hfq-binding activity. In other words, the Hfqbinding module must be located near the base-pairing region for efficient riboregulation.

\section{Design of synthetic Hfq-binding sRNAs}

We have demonstrated that the Hfq-binding module and the base-pairing region in Hfq-binding sRNAs can be separated by as much as $10 \mathrm{nt}$ without a significant loss of the silencing ability (Fig. 7). We showed previously that the 14-nt region of SgrS is sufficient for the stable base-pairing with the target $p t s G$ mRNA to execute efficient regulation (Maki et al. 2010). Thus, it is expected that if a sequence longer than $14 \mathrm{nt}$ 
A

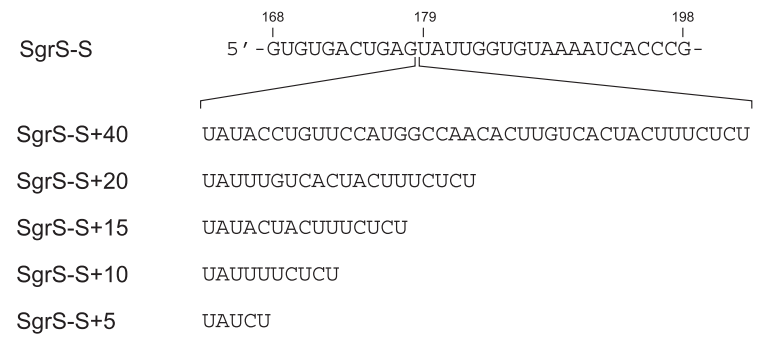

B

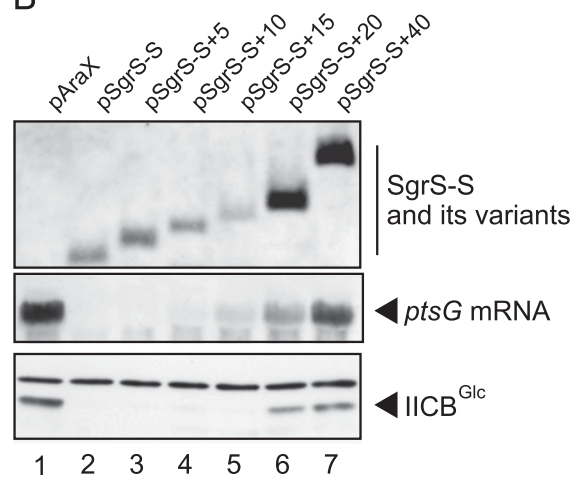

C

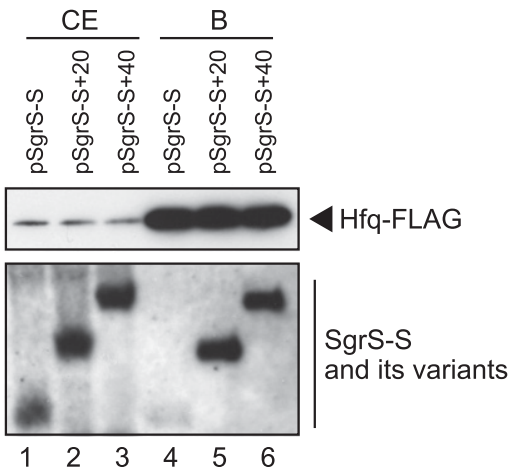

FIGURE 7. Effects of distance between base-pairing region and Hfq-binding module. $(A)$ Nucleotide sequences of SgrS-S and the spacing mutants. The inserted sequences are derived from the $g f p$ gene. $(B)$ Properties of SgrS-S variants. IT1568 cells harboring indicated plasmids were grown in the presence of $0.2 \%$ arabinose. Total RNAs and proteins were prepared. RNA samples were resolved by $10 \%$ polyacrylamide gel electrophoresis in the presence of $8 \mathrm{M}$ urea and subjected to Northern blot analysis using SgrS probe 2 and a $p t s G$ probe. The following amounts of RNAs were loaded: SgrS variants, $15 \mu \mathrm{g}$; pts $G$ mRNA, $15 \mu \mathrm{g}$. Protein samples equivalent to $0.04 \mathrm{~A}_{600}$ units were subjected to Western blot analysis. $(C) \mathrm{Hfq}$ binding of SgrS$\mathrm{S}$ variants. Crude extracts (CE) and bound fraction (B) were prepared from TM771 ( $\Delta$ sgrS $h f q-$ FLAG-cat) cells harboring indicated plasmids as described in Materials and Methods. The crude extracts $(200 \mu \mathrm{L})$ and the bound fraction $(20 \mu \mathrm{L})$ were treated with phenol, precipitated, and washed with ethanol. Each precipitate was dissolved in $10 \mu \mathrm{L}$ of RNA buffer $(0.02 \mathrm{M}$ sodium acetate at $\mathrm{pH} 5.5,0.5 \%$ SDS, and $1 \mathrm{mM}$ EDTA), resolved by $10 \%$ polyacrylamide gel electrophoresis in the presence of $8 \mathrm{M}$ urea and subjected to Northern blotting using SgrS probe 2.

complementary to the ribosome-binding site of any mRNA is connected to an Hfq-binding module within a spacing of 10 $\mathrm{nt}$, the resulting RNA molecule is able to silence the target mRNA efficiently. To test this prediction, we have constructed several plasmids expressing synthetic sRNAs in which sequences complementary either to $\operatorname{sodB}$, ompA, or ompF are placed immediately upstream of the Hfq-binding module of SgrS (Fig. 8A). The expression and the silencing ability of these synthetic sRNAs were examined by Northern blotting. As shown in Figure 8B, all of these sRNAs are stably expressed and repressed efficiently the expression of $\operatorname{sodB}, \operatorname{omp} A$, and $о m p F$ mRNA, respectively in $h \mathrm{fq}^{+}$but not $\Delta h f q$ backgrounds. Thus, we were able successfully to design synthetic Hfq-binding sRNAs to target desired mRNAs.

\section{DISCUSSION}

Hfq is essential for bacterial trans-acting sRNAs to regulate the translation and stability of target mRNAs. Studies on
SgrS have clarified the roles of $\mathrm{Hfq}$ in sRNA action. The primary role of Hfq is to facilitate the base-pairing between SgrS and the target $p t s G$ mRNA by accelerating the rate of duplex formation (Kawamoto et al. 2006). Another important role of Hfq is to recruit RNase $\mathrm{E}$ near $p t s G$ mRNA to destabilize the sRNA-mRNA hybrid (Morita et al. 2005). In addition, Hfq apparently stabilizes SgrS by protecting from the attack of ribonucleases because the abundance of SgrS is significantly low in cells lacking Hfq (Kawamoto et al. 2005; Maki et al. 2010). This view is supported also by the finding that levels of the SgrS variants decrease when $\mathrm{Hfq}$ binding is impaired riboregulation (Otaka et al. 2011). An important unsolved question is how Hfq promotes the base-pairing between SgrS and $p t s G$ mRNA. The identification of Hfq-binding sites on SgrS is certainly required for understanding the mechanism by which Hfq facilitates the base-pairing. We demonstrated in the previous study that the long terminator poly $(\mathrm{U})$ tail of sRNAs is essential for functional Hfq binding and therefore for riboregulation (Otaka et al. 2011).

In this study, we have unveiled the composition of the functional Hfq-binding site of bacterial sRNAs by starting to address the question of whether the Rhoindependent terminator is sufficient for Hfq binding. We demonstrated that the internal stem-loop preceded by a U-rich sequence of SgrS, in addition to the Rho-independent terminator with a long $\operatorname{poly}(\mathrm{U})$ tail, is crucial and sufficient for Hfq binding. Both in vivo and in vitro binding studies established that these two components are needed for the efficient binding of SgrS to Hfq that is a prerequisite for riboregulation. We conclude that the functional Hfqbinding module of SgrS consists of the internal hairpin preceded by a U-rich sequence and the terminator hairpin followed by a long $\operatorname{poly}(\mathrm{U})$ tail. We propose that this double hairpin structure represents one type of functional Hfq-binding module. In fact, the $3^{\prime}$ region of several known Hfq-binding sRNAs shares these features (Table 1). We also showed that the internal hairpin of SgrS is dispensable for Hfq action if a U-rich sequence is placed just before the terminator hairpin. Thus, the terminator hairpin preceded by a U-rich sequence and followed by a long poly $(\mathrm{U})$ tail is a simple type of functional Hfq-binding module. We noticed that many sRNAs possess a U-rich sequence just before the Rho-independent terminator (Table 1). We 
A

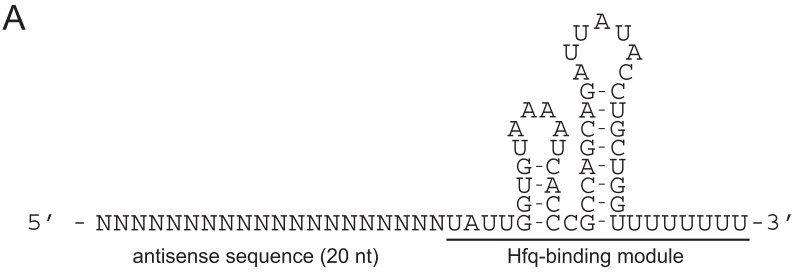

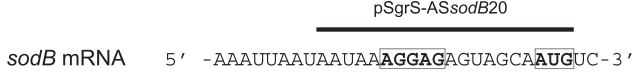

$$
\begin{aligned}
& \text { pSgrS-ASompA20 } \\
& \text { OmpA mRNA 5' -UUUGGAUGAUAACGAGGCGCAAAAAAUGAA- }{ }^{\prime} \\
& \text { pSgrS-ASompF20 }
\end{aligned}
$$

B

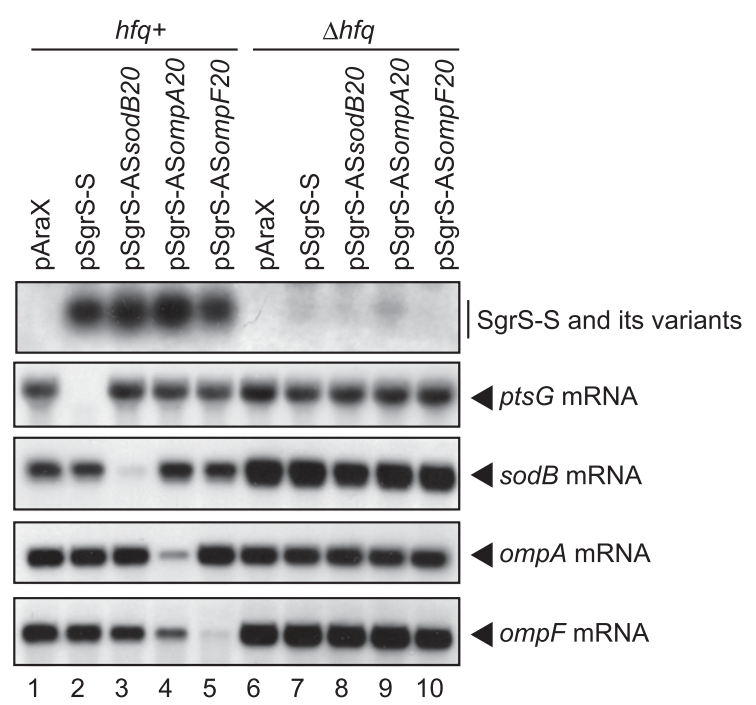

FIGURE 8. Design of synthetic Hfq-binding sRNAs. (A) Organization of synthetic Hfq-binding sRNAs. The Hfq-binding module is derived from SgrS. (N) The antisense sequence (20 nt) complementary to the translation initiation region of target mRNAs. Sequences of the translation initiation region of $s o d B$, ompA, or ompF mRNAs are shown below. The upper bars represent the sequences corresponding to the antisense sequences used. The Shine-Dalgarno sequences and the translation initiation codons are boxed. (B) Expression and silencing ability of synthetic Hfq-binding sRNAs. IT1568 $\left(h f q^{+}\right)$and TM589 $(\Delta h f q)$ cells harboring indicated plasmids were grown in LB medium containing $0.2 \%$ arabinose. Total RNAs were prepared. The RNA samples were subjected to Northern blot analysis using SgrS probe 2, a $p t s G$ probe, an $s o d B$ probe, an $o m p A$ probe, and an $o m p F$ probe. The following amounts of RNAs were loaded: SgrS-S variants, $5 \mu \mathrm{g}$; $p t s G$ mRNA, $10 \mu \mathrm{g}$; sodB mRNA, $5 \mu \mathrm{g}$; ompA mRNA, $1 \mu \mathrm{g}$; ompF mRNA, $1 \mu \mathrm{g}$.

conclude that the functional Hfq-binding module of bacterial sRNAs consists of either a double or single hairpin preceded by a U-rich sequence and followed by a 3 '-poly $(\mathrm{U})$ tail. A few sRNAs such as MicC and OmrA seem to lack an internal U-rich sequence near the terminator or internal hairpin structure. It will be interesting to study what else is needed for efficient Hfq binding, in addition to the Rhoindependent terminator, in these cases.
This study indicates that the Rho-independent transcription terminator is entirely included in the functional Hfqbinding module of sRNAs. In other words, the Hfq-binding module and Rho-independent transcription terminator cannot be separated from each other. On the other hand, the base-pairing region is structurally distinct from the Hfqbinding module, although it partially overlaps with the Hfq-binding module in SgrS. In fact, we showed that the basepairing region can be separated physically from the functional Hfq-binding module, although increasing the spacing between two elements gradually reduces the silencing ability (Fig. 7). Thus, we are now able to have a clear view concerning the structural organization of Hfq-binding sRNAs. They contain at least one base-pairing region and the 3' Hfa-binding module including the Rho-independent transcription terminator. Based on this view, we were able to successfully design synthetic Hfq-binding sRNAs to target desired mRNAs (Fig. 8).

It is highly possible that the Hfq binds the poly(U) tail of sRNAs through its proximal side; a recent structural study demonstrated that the proximal RNA-binding site of Hfq prefers to accommodate U-rich RNA 3' ends (Sauer and Weichenrieder 2011). This study showed that the internal stem-loop structure along with the preceding U-rich sequence is also required for the efficient Hfq binding of SgrS. An intriguing question is whether a single Hfq hexamer binds simultaneously to the terminator $\operatorname{poly}(\mathrm{U})$ tail and to the internal stem-loop/U-rich sequence or two sites are recognized by two different Hfq hexamers. The observation that Hfq forms a stable 1:1 complex with SgrS favors the view that a single $\mathrm{Hfq}$ hexamer binds simultaneously to the terminator poly $(\mathrm{U})$ tail and the internal stem-loop/U-rich sequence. If this were the case, it would be possible that the proximal side of $\mathrm{Hfq}$ binds the terminator poly $(\mathrm{U})$ tail, while the internal stem-loop/U-rich sequence could interact with the distal side or some other portion of the same Hfq molecule. In fact, it was demonstrated recently that a U-rich RNA oligonucleotide corresponding to the internal Hfq-binding site of DsrA interacts with not only the proximal site but also the distal site and the rim of the $\mathrm{Hfq}$ hexamer (Wang et al. 2011). An alternative possibility would be that the poly $(\mathrm{U})$ tail of one SgrS molecule is recognized by the proximal side of one Hfq hexamer, while the internal stem-loop/U-rich sequence binds the distal side of another $\mathrm{Hfq}$ hexamer as proposed recently for DsrA-Hfq interaction (Wang et al. 2011). Structural studies on the Hfq-functional sRNA complexes are certainly required to clarify why the hairpin structure is needed for the efficient Hfq binding and how exactly $\mathrm{Hfq}$ binds to the Hfq-binding modules to facilitate base-pairing between sRNAs and target mRNAs.

The central role of Hfq in sRNA-mediated gene regulation is to facilitate the base-pairing between sRNAs and their target mRNAs. Two possible mechanisms by which Hfq promotes the interaction between sRNAs and their targets have been described (Storz et al. 2004; Valentin-Hansen 
TABLE 1. Sequences of the $3^{\prime}$ region of Hfq-binding sRNAs

\begin{tabular}{|c|c|}
\hline SRNA & Seque \\
\hline Sgrs & -GUAUUGGUGUAAAAUCACCCGCCAGCAGAUUAUACCUGCUGGUUUUUUUU \\
\hline $\mathrm{MicF}$ & -AUUUAUUACCGUCAUUCAUUUCUGAAUGUCUGUUUACCCQUAUUUCAACCGGAUGCCUCGCAUUCGGUUUUUUUU \\
\hline DsrA & -AUUUUUUAAGUGCUUCUUGCUUAAGCAAGUUUCAUCCCGACCCCCUCAGGGUCGGGAUUUUUUU \\
\hline Spot42 & -AUUUGGCUGAAUAUUUUAGCCGCCCCAGUCAGUAAUGACUGGGGCGUUUUUUAUU \\
\hline RybB & -AUUUUGUGGAGCCCAUCAACCCCGCCAUUUCGGUUCAAGGUUGAUGGGUUUUUUGUU \\
\hline ArcZ & -GUGUUGGCGCAGUAUUCGCGCACCCCGGUCUAGCCGGGGUCAUUUUUU \\
\hline MgrR & -CUGUUAGCGUAAAAGCAAAACACAAAUCUAUCCAUGCAAGCAUUCACCGCCGGUUUACUGGCGGUUUUUUUU \\
\hline RyhB & -GUAUUACUUAGCCAGCCGGGUGCUGGCUUUUUUUUU \\
\hline RseX & -A[UUUUUUGGGCCGGCAUGAUGCCGGCUUUUUUUU \\
\hline OxyS & -cUUUUGCGGAUCUCCAGGAUCCGCUUUUUUUUU \\
\hline GevB & -AUUAAUGUAGCACCGCCUAAUUGCGGTGCUUUUUUUU \\
\hline RprA & -GUCUUUGGCCCAUCUCCCACGAUGGGCUUUUUUUU \\
\hline GlmZ & -cUGUUUUAACGCCUGCUCCGUAAUAAGAGCAGGCGUUUUUUU \\
\hline ChiX & -CUCUUUGACGGGCCAAUAGCGAUAUUGGCCAUUUUUUU \\
\hline MicA & -AUUUUGGCCACUCACGAGUGGCCUUUUUCUUUU \\
\hline RydC & -GUAUUCUUCGCCUGUACCACGGGUCGGUUUUAGUACAGGCGUUUUCUUU \\
\hline CyaR & -CUCCUUAGCCUGUGUAAUCUCCCUUACACGGGCUUAUUUUUU \\
\hline MicC & -CCAUUGCAUUGCCACUGAUUUUCCAACAUAUAAAAAGACAAGCCCGAACAGUCGUCCGGGCUUUUUUUUU \\
\hline OmrA & -UCUUCGUACCCUGUCUCUUGCACCAACCUGCGCGGAUGCGCAGGUUUUUUUU \\
\hline OmrB & -GUUGAGCACAUGAAUUACACCAGCCUGCGCAGAUGCGCAGGUUUUUUUU \\
\hline
\end{tabular}

RNA sequences transcribed from corresponding genes (http://www.genome.jp/kegg/) were shown assuming that transcription termination occurs at the last $U$ within the polyU tail. Sequences of predicted Rho-independent terminator are italicized. Sequences corresponding to internal hairpin are shadowed, while U-rich sequences before internal and/or terminator hairpins are boxed. et al. 2004). First, Hfq may unfold the secondary structure of sRNAs and/or target mRNAs to expose the base-pairing regions by acting as an RNA chaperone. In this case, the primary role of $\mathrm{Hfq}$ is to relieve structural restrictions in the base-pairing regions of sRNAs and/or mRNAs. The second mechanism would be that Hfq acts by increasing the local concentrations of the RNAs involved in base-pairing through simultaneous binding to both sRNAs and target mRNAs. Although these two models are not mutually exclusive, the recent studies are consistent with a view that unfolding or remodeling of sRNAs/mRNAs could be at least an important mechanism in general by which Hfq facilitates the base-pairing between sRNAs and their target mRNAs. For example, it was shown that $\mathrm{Hfq}$ relieves negative interactions within the rpoS leader, a target for positive regulation by DsrA (Soper and Woodson 2008). We found that a 14-nt RNA oligo corresponding to the minimum base-pairing region of SgrS is able to inhibit $p t s G$ translation without the help of $\mathrm{Hfq}$ in vitro (Maki et al. 2010). More recently, it has been shown that a truncated RyhB in which internal portions other than the base-pairing region are largely deleted is able to repress efficiently the target $\operatorname{sodB}$ mRNA in the absence of Hfq in vivo (Hao et al. 2011).

\section{MATERIALS AND METHODS}

\section{Bacterial strains and plasmids}

The E. coli K12 strains and plasmids used in this study are listed in Table 2. The DNA primers used in this study are listed in Supplemental Table S1. Plasmids pQESgrS1 and pQESgrS14, derivatives of $\mathrm{pQE} 80 \mathrm{~L}$, are described by Kawamoto et al. (2006) and Maki et al. (2010). Plasmid pQESgrS14R was constructed as follows: pQESgrS14 was used to amplify DNA fragment 1 containing the mutated sgrS region $(-2$ to +210$)$ with primers 401 and 1077. Similarly, DNA fragment 2 containing the mutated sgrS region $(+181$ to +247$)$ was amplified with primers 402 and 1076. Then, the DNA fragments 1 and 2 were used to amplify the mutated sgrS region $(-2$ to +247$)$, in which TCACC between +192 and +196 is changed to CGTGG with primers 401 and 402. The amplified fragment was digested with EcoRI and HindIII and cloned into pQE80L. pAraS, a derivative of pMW218, was used as a vector to construct a series of $\mathrm{pSgrS}$-series plasmids.

pSgrS14a was constructed as follows: pSgrS was used to amplify DNA fragment 3 containing the mutated $s g r S$ region $(+1$ to +200 ) with primers 928 and 1008. Similarly, DNA fragment 4 containing the mutated sgrS region $(+172$ to +229$)$ was amplified with primers 930 and 1007. Then, the DNA fragments 3 and 4 were used to amplify the mutated sgrS region $(+1$ to +229$)$, in which GGTGT between +183 and +187 is changed to CCACG with primers 928 and 930. The amplified fragment was digested with SalI and HindIII and cloned into pAraS to obtain pSgrS14a.

pSgrS14aR was constructed as follows: pSgrS14a was used to amplify DNA fragment 5 containing the mutated $\operatorname{sgrS}$ region $(+1$ to +210 ) with primers 928 and 1077. Similarly, DNA fragment 6 containing the mutated $s g r S$ region $(+181$ to +229$)$ was amplified with primers 930 and 1076. Then, the DNA fragments 5 and 6 were used to amplify the mutated $\operatorname{sgrS}$ region $(+1$ to +229$)$, in which GGTGTAAAATCACC between +183 and +196 is changed to CCACGAAAACGTGG with primers 928 and 930. The amplified fragment was digested with SalI and HindIII and cloned into pAraS to obtain pSgrS14aR.

pSgrS14b was constructed as follows: pSgrS was used to amplify DNA fragment 7 containing the mutated sgrS region $(+1$ to +197$)$ with primers 928 and 1029. Similarly, DNA fragment 8 containing the mutated $s g r S$ region $(+173$ to +229$)$ was amplified with primers 930 and 1028. Then, the DNA fragments 7 and 8 were used to amplify the mutated $s g r S$ region $(+1$ to +229$)$, in which GGTGT between +183 and +187 is changed to TAGCA with primers 928 and 930. The amplified fragment was digested with SalI and HindIII and cloned into pAraS to obtain pSgrS14b.

Other pSgrS-series plasmids were constructed as follows: A DNA fragment was amplified with 928 primer and each of the reverse primers indicated in Supplemental Table S1. The amplified fragment was digested with SalI and HindIII and cloned into pAraS. To construct pTSgrS-series plasmids, the EcoRI and HindIII fragment containing each of the sgrS variants derived from pSgrS-series plasmids was cloned into pTWV228.

pSgrS-S series plasmids were constructed as follows: A DNA fragment containing the sgrS region with extra sequences was amplified with 929 primer and each of the forward primers 
Ishikawa et al.

TABLE 2. Bacterial strains and plasmids used in this study

\begin{tabular}{|c|c|c|}
\hline Strain/plasmid & Relevant genotype and property & Source \\
\hline \multicolumn{3}{|l|}{ Strain } \\
\hline IT1568 & W3110m/c & Laboratory stock \\
\hline TM589 & W3110m/c $\Delta h f q$ & Morita et al. 2005 \\
\hline TM771 & W3110m/c hfq-FLAG-cat $\Delta$ (sgrR-sgrS) & Otaka et al. 2011 \\
\hline \multicolumn{3}{|c|}{ 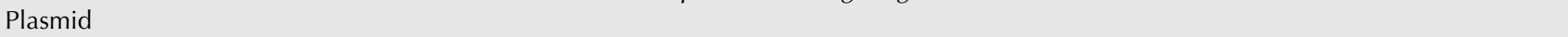 } \\
\hline pQE80L & High-copy-number plasmid vector & QIAGEN \\
\hline pQESgrS1 & Derivative of pQE80L carrying sgrS & Kawamoto et al. 2006 \\
\hline pQESgrS14 & Derivative of pQE80L carrying sgrS14 & Maki et al. 2010 \\
\hline pQESgrS14R & Derivative of $\mathrm{pQE} 80 \mathrm{~L}$ carrying sgrS14R & This study \\
\hline pQE-Hfq-His & Derivative of pQE80L carrying $h \mathrm{fq}-\mathrm{His}_{6}$ & Kawamoto et al. 2006 \\
\hline pMW218 & Low-copy-number plasmid vector & NIPPON GENE \\
\hline pAraS & Derivative of pMW218 carrying araC-PBAD & Otaka et al. 2011 \\
\hline pSgrS & Derivative of pAraS carrying sgrS & Otaka et al. 2011 \\
\hline pSgrS14a & Derivative of pAraS carrying sgrS14a & This study \\
\hline pSgrS14aR & Derivative of pAraS carrying sgrS14aR & This study \\
\hline pSgrS14b & Derivative of pAraS carrying sgrS14b & This study \\
\hline pSgrS14bR & Derivative of pAraS carrying sgrS14bR & This study \\
\hline pSgrSLS4U & Derivative of pAraS carrying sgrS-LS4U & Otaka et al. 2011 \\
\hline pSgrSLGC & Derivative of pAraS carrying sgrSLGC & This study \\
\hline pSgrS182C & Derivative of pAraS carrying sgrS182C & This study \\
\hline pSgrSCCGC & Derivative of pAraS carrying sgrSCCGC & This study \\
\hline pSgrSi & Derivative of pAraS carrying sgrS+UAUU & This study \\
\hline pSgrSiCCGC & Derivative of pAraS carrying sgrS+CCGC & This study \\
\hline pSgrSiAAAA & Derivative of pAraS carrying sgrS $+A A A A$ & This study \\
\hline pSgrSiUUUU & Derivative of pAraS carrying sgrS+UUUU & This study \\
\hline pSgrS14aUUUU & Derivative of pAraS carrying sgrS14aUUUU & This study \\
\hline pSgrS14aAAAA & Derivative of pAraS carrying sgrS14aAAAA & This study \\
\hline pSgrS14aCCCC & Derivative of pAraS carrying sgrS14aCCCC & This study \\
\hline pSgrS $\Delta 14$ & Derivative of pAraS carrying sgrSs 14 & This study \\
\hline pSgrS $\Delta 10$ & Derivative of pAraS carrying sgrS $\Delta 10$ & This study \\
\hline $\mathrm{pSgrS} \Delta 5$ & Derivative of pAraS carrying sgrS $\Delta 5$ & This study \\
\hline pTWV228 & High-copy-number plasmid vector & TAKARA \\
\hline pTSgrS14a & Derivative of pTWV carrying sgrS14a & This study \\
\hline pTSgrS14b & Derivative of pTWV carrying sgrS14b & This study \\
\hline pTSgrSCCGC & Derivative of pTWV carrying sgrSCCGC & This study \\
\hline pAraX & Derivative of pMW218 carrying araC-PBAD & Otaka et al. 2011 \\
\hline pSgrS-S & Derivative of pAraX carrying sgrS-S & Otaka et al. 2011 \\
\hline pSgrS-S+5 & Derivative of pAraX carrying sgrS-S+5 & This study \\
\hline pSgrS-S+10 & Derivative of pAraX carrying sgrS-S+10 & This study \\
\hline pSgrS-S+15 & Derivative of pAraX carrying sgrS-S+15 & This study \\
\hline pSgrS-S+20 & Derivative of pAraX carrying sgrS-S+20 & This study \\
\hline pSgrS-S+40 & Derivative of pAraX carrying sgrS-S+40 & This study \\
\hline pSgrS-ASsodB20 & Derivative of pAraX carrying sgrS-ASsodB20 & This study \\
\hline pSgrS-ASompA20 & Derivative of pAraX carrying sgrS-ASompA2O & This study \\
\hline pSgrS-ASompF20 & Derivative of pAraX carrying sgrS-ASompF2O & This study \\
\hline
\end{tabular}

indicated in Supplemental Table S1. The amplified fragment was digested with XbaI and HindIII and cloned into pAraX.

pSgrS-AS series plasmids were constructed as follows: A DNA fragment containing the sgrS region with extra sequences was amplified with 929 primer and each of the forward primers indicated in Supplemental Table S1. The amplified fragment was digested with XbaI and HindIII and cloned into pAraX.

\section{Northern blotting}

Cells carrying the sRNA-expressing plasmids were grown at $37^{\circ} \mathrm{C}$ to mid-log phase in LB medium supplemented with ampicillin (50 $\mu \mathrm{g} / \mathrm{mL})$, kanamycin $(15 \mu \mathrm{g} / \mathrm{mL})$, and indicated amounts of IPTG or arabinose. Total RNAs were isolated as described (Aiba et al. 1981). RNA samples were resolved by $1.2 \%$ agarose gel electrophoresis in the presence of formaldehyde unless specified and blotted onto Hybond- $\mathrm{N}^{+}$membrane (Amersham Biosciences). The RNAs were visualized using digoxigenin (DIG) reagents and kits for non-radioactive nucleic acid labeling and a detection system (Roche Molecular Biochemicals) according to the procedure specified by the manufacturer. The following DIG-labeled DNA probes were prepared by PCR using DIG-dUTP: a 305-bp fragment corresponding to the $5^{\prime}$ region of $p t s G$ (ptsG probe); a 150-bp fragment containing the $5^{\prime}$ portion $(+1$ to +150$)$ of SgrS (SgrS probe 1$)$; 
a 55-bp fragment containing the $3^{\prime}$ portion $(+183$ to +227$)$ of SgrS (SgrS probe 2); a 210-bp fragment corresponding to the $5^{\prime}$ region of $\operatorname{sodB}$ ( $\operatorname{sodB}$ probe); a 235-bp fragment corresponding to the $5^{\prime}$ region of ompA (ompA probe); and a 220-bp fragment corresponding to the $5^{\prime}$ region of ompF (ompF probe).

\section{Western blotting}

Cells carrying the sRNA-expressing plasmids were grown at $37^{\circ} \mathrm{C}$ to mid-log phase in LB medium supplemented with ampicillin (50 $\mu \mathrm{g} / \mathrm{mL})$, kanamycin $(15 \mu \mathrm{g} / \mathrm{mL})$, and indicated amounts of IPTG or arabinose. The cultures $(1 \mathrm{~mL})$ were centrifuged, and the cell pellets were suspended in $100 \mu \mathrm{L}$ of SDS-PAGE loading buffer (62.5 mM Tris- $\mathrm{HCl}$ at $\mathrm{pH}$ 6.8, 2\% SDS, 10\% glycerol, 5\% $\beta$-mercaptoethanol, $0.1 \%$ Bromophenol Blue). The sample was heated for $5 \mathrm{~min}$ at $100^{\circ} \mathrm{C}$ and subjected to a $12 \%$ (for IICB ${ }^{\text {Glc }}$ ) or $15 \%$ (for Hfq-Flag) polyacrylamide- $0.1 \%$ SDS gel electrophoresis and transferred to an Immobilon membrane (Millipore). The membranes were treated either with an anti-Flag monoclonal antibody (Sigma-Aldrich) or anti-IIB ${ }^{\text {Glc }}$ polyclonal antibodies (Tanaka et al. 2000). Signals were visualized by the Lumi-light Western Blotting Substrate (Roche).

\section{Pull-down assay}

TM771 ( $\Delta$ sgrS $h f q$-FLAG-cat) cells harboring the indicated plasmids were grown in $100 \mathrm{~mL}$ of $\mathrm{LB}$ medium to $\mathrm{A}_{600}=0.6$ in the presence of $0.4 \%$ (for cells harboring low-copy plasmids) or $0.1 \%$ (for cells harboring high-copy plasmids) arabinose. Cells were harvested and washed with $10 \mathrm{~mL}$ of STE buffer $(100 \mathrm{mM} \mathrm{NaCl}$, $10 \mathrm{mM}$ Tris- $\mathrm{HCl}$ at $\mathrm{pH} 8.0,1 \mathrm{mM}$ EDTA). The cell pellets were suspended in $12 \mathrm{~mL}$ of ice-cold IP buffer $(20 \mathrm{mM}$ Tris- $\mathrm{HCl}$ at $\mathrm{pH}$ 8.0, $0.1 \mathrm{M} \mathrm{KCl}, 5 \mathrm{mM} \mathrm{MgCl}_{2}, 10 \%$ glycerol, $0.1 \%$ Tween 20 ). The cell suspension was sonicated and centrifuged at $10,000 \mathrm{~g}$ for 30 $\min$ at $4^{\circ} \mathrm{C}$. The supernatant (crude extract) was incubated with $50 \mu \mathrm{L}$ of anti-Flag M2-agarose suspension (Sigma-Aldrich) for $30 \mathrm{~min}$ at $4^{\circ} \mathrm{C}$. The mixture was filtered by using a mini chromatography column (Bio-Rad). The agarose beads were washed with $10 \mathrm{~mL}$ of IP buffer twice. The proteins bound to the beads were eluted with $50 \mu \mathrm{L}$ of IP buffer containing $0.4 \mathrm{mg} / \mathrm{mL}$ Flag peptide (Sigma-Aldrich) and used as the bound fraction (B). The samples were analyzed by Western blotting. To analyze RNAs, the crude extract $(10 \mu \mathrm{L})$ and the bound fraction $(10 \mu \mathrm{L})$ were treated with phenol, precipitated, and washed with ethanol. Each precipitate was dissolved in $20 \mu \mathrm{L}$ of RNA buffer $(0.02 \mathrm{M}$ sodium acetate at $\mathrm{pH} 5.5,0.5 \%$ SDS, and $1 \mathrm{mM}$ EDTA). The following amounts of RNA samples were subjected to Northern blotting: crude extract (CE), $3 \mu \mathrm{L}$; bound fraction (B), $3 \mu \mathrm{L}$.

\section{Preparation of RNAs in vitro}

SgrS variants were synthesized by in vitro transcription using T7 RNA polymerase and PCR-amplified DNA templates, containing T7 promoter and the transcribed sequences corresponding to SgrS variants. In vitro transcription reaction were performed by using a CUGA7 in vitro transcription kit (Nippon Genetech) according to the manufacturer's instruction. $\left[\alpha-{ }^{32} \mathrm{P}\right] \mathrm{UTP}$ (PerkinElmer Life and Analytical Sciences) was added to the reaction mixture to generate ${ }^{32} \mathrm{P}$-labeled SgrS RNA. The DNA templates for SgrS, SgrS14b, SgrS14bR, and SgrSCCGC were amplified from pSgrS,
pSgrS14b, pSgrS14bR, and pSgrSCCGC, respectively, with primers 1330 and 1332. The DNA template for SgrS4U was amplified from pSgrS4U with primers 1330 and 1333. Primer 1330 contains the promoter sequence for T7 RNA polymerase. Primers 1332 and 1333 are designed to create the DNA templates in which the $3^{\prime}$ ends of transcribed sequences correspond to $8 \mathrm{U}$ and $4 \mathrm{U}$, respectively. The PCR products were treated with phenol and precipitated with ethanol. The precipitated DNAs were dissolved in 10-20 $\mu \mathrm{L}$ of $\mathrm{H}_{2} \mathrm{O}$. The RNA transcripts were treated with phenol and precipitated with ethanol. The precipitated RNAs were dissolved in $50 \mu \mathrm{L}$ of $\mathrm{H}_{2} \mathrm{O}$. The concentration of RNA was determined by gel electrophoresis followed by ethidium bromide staining.

\section{Gel mobility shift assay}

${ }^{32}$ P-labeled SgrS RNA (0.2 pmol) and indicated amounts of purified $\mathrm{Hfq}^{-\mathrm{His}_{6}}$ were mixed in $5 \mu \mathrm{L}$ of binding buffer $(20$ $\mathrm{mM}$ Tris- $\mathrm{HCl}$ at $\mathrm{pH} 8.0,1 \mathrm{mM} \mathrm{DTT}, 1 \mathrm{mM} \mathrm{MgCl} 2,20 \mathrm{mM} \mathrm{KCl}$, $10 \mathrm{mM} \mathrm{Na} \mathrm{H} \mathrm{PO}_{4}-\mathrm{NaH}_{2} \mathrm{PO}_{4}$ at $\mathrm{pH}$ 8.0) containing $1 \mu \mathrm{g}$ of yeast tRNA (Invitrogen Japana). Hfq-His ${ }_{6}$ was purified as described previously (Maki et al. 2010). An excess amount (1 or 2 pmol) of each unlabeled SgrS variant was added together for competition experiments. The mixtures were incubated for $15 \mathrm{~min}$ at $37^{\circ} \mathrm{C}$, and $5 \mu \mathrm{L}$ of loading buffer (25\% glycerol, $0.05 \%$ Bromophenol Blue) was added to the reaction mixtures. The samples were loaded on a $4 \%$ polyacrylamide gel in $0.5 \times$ TBE containing $5.0 \%$ glycerol. The electrophoresis was performed at $4^{\circ} \mathrm{C}$. After electrophoresis, the gel was dried up and subjected to autoradiography.

\section{SUPPLEMENTAL MATERIAL}

Supplemental material is available for this article.

\section{ACKNOWLEDGMENTS}

We thank Susan Gottesman for comments on the manuscript. This work was supported by Grants-in-Aid from the Ministry of Education, Culture, Sports, Science and Technology of Japan (to H.A. and T.M.).

Received November 28, 2011; accepted January 24, 2012.

\section{REFERENCES}

Aiba H. 2007. Mechanism of RNA silencing by Hfq-binding small RNAs. Curr Opin Microbiol 10: 134-139.

Aiba H, Adhya S, de Crombrugghe B. 1981. Evidence for two functional gal promoters in intact Escherichia coli cells. J Biol Chem 256: 11905-11910.

Aiba H, Hanamura A, Yamano H. 1991. Transcriptional terminator is a positive regulatory element in the expression of the Escherichia coli crp gene. J Biol Chem 266: 1721-1727.

Balbontin R, Fiorini F, Figueroa-Bossi N, Casadesus J, Bossi L. 2010. Recognition of heptameric seed sequence underlies multi-target regulation by RybB small RNA in Salmonella enterica. Mol Microbiol 78: 380-394.

Brescia CC, Mikulecky PJ, Feig AL, Sledjeski DD. 2003. Identification of the Hfq-binding site on DsrA RNA: Hfq binds without altering DsrA secondary structure. RNA 9: 33-43. 
Geissmann TA, Touati D. 2004. Hfq, a new chaperoning role: Binding to messenger RNA determines access for small RNA regulator. EMBO J 23: 396-405.

Gottesman S. 2004. The small RNA regulators of Escherichia coli: Roles and mechanisms ${ }^{\star}$. Annu Rev Microbiol 58: 303-328.

Gottesman S, Storz G. 2010. Bacterial small RNA regulators: Versatile roles and rapidly evolving variations. Cold Spring Harb Perspect Biol 1: 1-16.

Hao Y, Zhang ZJ, Erickson DW, Huang M, Huang Y, Li J, Hwa T, Shi H. 2011. Quantifying the sequence-function relation in gene silencing by bacterial small RNAs. Proc Natl Acad Sci 108: 12473-12478.

Kawamoto H, Morita T, Shimizu A, Inada T, Aiba H. 2005. Implication of membrane localization of target mRNA in the action of a small RNA: Mechanism of post-transcriptional regulation of glucose transporter in Escherichia coli. Genes Dev 19: 328-338.

Kawamoto H, Koide Y, Morita T, Aiba H. 2006. Base-pairing requirement for RNA silencing by a bacterial small RNA and acceleration of duplex formation by Hfq. Mol Microbiol 61: 1013-1022.

Link TM, Valentin-Hansen P, Brennan RG. 2009. Structure of Escherichia coli Hfq bound to polyriboadenylate RNA. Proc Natl Acad Sci 106: 19292-19297.

Maki K, Morita T, Otaka H, Aiba H. 2010. A minimal base-pairing region of a bacterial small RNA SgrS required for translational repression of ptsG mRNA. Mol Microbiol 76: 782-792.

Moller T, Franch T, Hojrup P, Keene DR, Bachinger HP, Brennan RG, Valentin-Hansen P. 2002. Hfq: A bacterial Sm-like protein that mediates RNA-RNA interaction. Mol Cell 9: 23-30.

Morita T, Maki K, Aiba H. 2005. RNase E-based ribonucleoprotein complexes: Mechanical basis of mRNA destabilization mediated by bacterial noncoding RNAs. Genes Dev 19: 2176-2186.

Otaka H, Ishikawa H, Morita T, Aiba H. 2011. PolyU tail of rhoindependent terminator of bacterial small RNAs is essential for Hfq action. Proc Natl Acad Sci 108: 13059-13064.

Rasmussen AA, Eriksen M, Gilany K, Udesen C, Franch T, Petersen C, Valentin-Hansen P. 2005. Regulation of $o m p A$ mRNA stability:
The role of a small regulatory RNA in growth phase-dependent control. Mol Microbiol 58: 1421-1429.

Sauer E, Weichenrieder O. 2011. Structural basis for RNA 3 '-end recognition by Hfq. Proc Natl Acad Sci 108: 13065-13070.

Schumacher MA, Pearson RF, Moller T, Valentin-Hansen P, Brennan RG. 2002. Structures of the pleiotropic translational regulator $\mathrm{Hfq}$ and an Hfq-RNA complex: A bacterial Sm-like protein. EMBO J 21: 3546-3556.

Soper TJ, Woodson SA. 2008. The rpoS mRNA leader recruits Hfq to facilitate annealing with DsrA sRNA. RNA 14: 1907-1917.

Storz G, Gottesman S. 2006. Versatile roles of small RNA regulators in bacteria. Cold Spring Harbor Laboratory Press, Cold Spring Harbor, NY.

Storz G, Opdyke JA, Zhang A. 2004. Controlling mRNA stability and translation with small, noncoding RNAs. Curr Opin Microbiol 7: 140-144.

Tanaka Y, Kimata K, Aiba H. 2000. A novel regulatory role of glucose transporter of Escherichia coli: Membrane sequestration of a global repressor Mlc. EMBO J 19: 5344-5352.

Valentin-Hansen P, Eriksen M, Udesen C. 2004. The bacterial Sm-like protein Hfq: A key player in RNA transactions. Mol Microbiol 51: $1525-1533$

Vanderpool CK, Gottesman S. 2004. Involvement of a novel transcriptional activator and small RNA in post-transcriptional regulation of the glucose phosphoenolpyruvate phosphotransferase system. Mol Microbiol 54: 1076-1089.

Vogel J, Luisi BF. 2011. Hfq and its constellation of RNA. Nat Rev Microbiol 9: 578-589.

Wang W, Wang L, Zou Y, Zhang J, Gong Q, Wu J, Shi Y. 2011. Cooperation of Escherichia coli Hfq hexamers in DsrA binding. Genes Dev 25: 2106-2117.

Zhang A, Wassarman KM, Ortega J, Steven AC, Storz G. 2002. The Sm-like Hfq protein increases OxyS RNA interaction with target mRNAs. Mol Cell 9: 11-22.

Zuker M. 2003. Mfold web server for nucleic acid folding and hybridization prediction. Nucleic Acids Res 31: 3406-3415. 

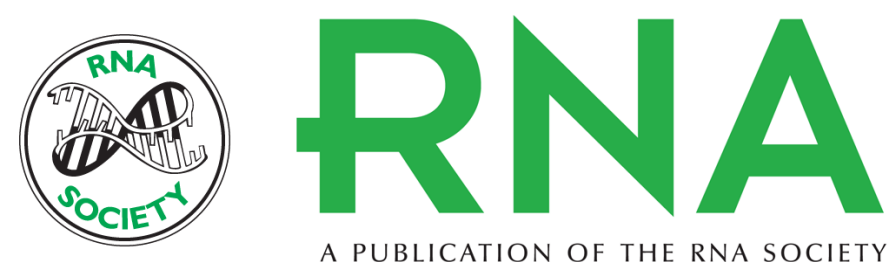

A PUBLICATION OF THE RNA SOCIETY

\section{The functional Hfq-binding module of bacterial sRNAs consists of a double or single hairpin preceded by a U-rich sequence and followed by a 3' poly(U) tail}

Hirokazu Ishikawa, Hironori Otaka, Kimika Maki, et al.

RNA 2012 18: 1062-1074 originally published online March 27, 2012

Access the most recent version at doi:10.1261/rna.031575.111

Supplemental Material

References

License

Email Alerting Service
http://rnajournal.cshlp.org/content/suppl/2012/02/23/rna.031575.111.DC1

This article cites 28 articles, 14 of which can be accessed free at: http://rnajournal.cshlp.org/content/18/5/1062.full.html\#ref-list-1

Receive free email alerts when new articles cite this article - sign up in the box at the top right corner of the article or click here.

To subscribe to RNA go to:

http://rnajournal.cshlp.org/subscriptions 